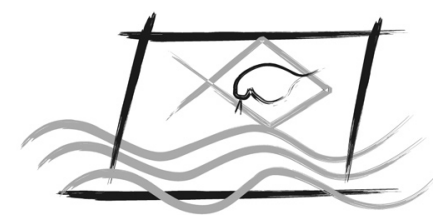

J. Braz. Soc. Ecotoxicol., v. 7, n. 1, 2012, 15-20

doi: $10.5132 / j b s e .2012 .01 .003$

ECOTOX - BRASIL

\title{
Potencial de espécies vegetais nativas do pólo industrial de Camaçari (BA, Brasil) como acumuladoras de elementos químicos contidos na poluição atmosférica
}

\author{
O.M. Santos, M.L.S. Guedes \& C.V.M. AraúJo \\ Universidade Federal da Bahia, Departamento de Botânica do Instituto de Biologia, Campus Universitário \\ de Ondina, CEP 40.170-115, Salvador, Bahia, Brasil.
}

(Received May 14, 2009; Accept June 13, 2011)

\begin{abstract}
Resumo
O presente estudo objetivou avaliar o potencial das espécies Anacardium occidentale L., Miconia albicans (Sw.) Triana e Byrsonima sericea DC. como acumuladores de alumínio (Al), arsênio (As), cobre $(\mathrm{Cu})$, níquel (Ni), zinco ( $\mathrm{Zn})$ e enxofre (S), provenientes das emissões atmosféricas do Pólo Industrial de Camaçari (PIC). As concentrações dos elementos foram determinadas nos tecidos foliares de três indivíduos adultos por espécie. Os dados mostraram que M. albicans é uma espécie acumuladora de $\mathrm{Al}, \mathrm{Cu}$ e S. Esta espécie apresentou sintomas de danos causados pela poluição atmosférica com cloroses e necroses sobre as folhas. Por outro lado, em $A$. occidentale e $B$. sericea a acumulação dos poluentes nos tecidos foliares foi, via de regra, menor. Em geral, os teores de $\mathrm{Zn}$ foram semelhantes em todas as espécies, enquanto as concentrações de As e $\mathrm{Ni}$ apresentaram valores abaixo do limite de quantificação. M. albicans demonstrou ser uma espécie com potencial para ser utilizada como biomonitor da poluição atmosférica no PIC.
\end{abstract}

Palavras-chave: Anacardium occidentale, Byrsonima sericea, Miconia albicans, poluição atmosférica

\section{Potential of native plant species from the Industrial Complex of Camaçari (Ba, Brazil) as accumulators of chemical elements contained in air pollution}

\begin{abstract}
The present study aimed to assess the potential of plant species Anacardium occidentale L., Miconia albicans (Sw.) Triana and Byrsonima sericea DC. as bioaccumulators of aluminum (Al), arsenic (As), copper ( $\mathrm{Cu})$, nickel (Ni), zinc ( $\mathrm{Zn})$ and sulphur (S), coming from industrial emission from the Industrial Complex of Camaçari (ICC). Element concentrations were measured in the leaves of three adult individuals per species. The data showed that M. albicans is an accumulator species of $\mathrm{Al}, \mathrm{Cu}$ and $\mathrm{S}$. Moreover, this specie showed visible damage symptoms on the leaves such as chloroses and necroses, which may be attributed to air pollution. On the other hand, the accumulation of the elements was lower in the leaves of $A$. occidentale and $B$. sericea. In general, $\mathrm{Zn}$ concentrations were similar in all species. As and $\mathrm{Ni}$ concentrations in the species were below detection limit. The results indicate that $M$. albicans may potentially be employed in biomonitoring studies in this region.
\end{abstract}

Keywords: Anacardium occidentale, Byrsonima sericea, Miconia albicans, air pollution 


\section{INTRODUÇÃO}

O maior complexo industrial integrado da América do Sul, o Pólo Industrial de Camaçari (PIC), localizado a cerca de $55 \mathrm{~km}$ da cidade do Salvador, Bahia, começou a operar desde 1978, estando hoje com uma área de aproximadamente $233 \mathrm{~km}^{2}$, distribuída entre os municípios de Camaçari e Dias D’Ávila. Apresenta mais de 60 indústrias de diversas áreas: alimento, automotiva, cervejaria, cimento, fertilizantes, metalúrgicas, petroquímica, plástico, química, resina entre outras (Lima et al., 2000; Almeida et al., 2003; Klumpp et al., 2003). Apenas em 1994 iniciou-se um programa de monitoramento do ar pela CETREL Proteção Ambiental nas áreas urbanizadas e circunvizinhas ao PIC, registrando material particulado, dióxido de enxofre, óxidos de nitrogênio, monóxido de carbono, ozônio, compostos orgânicos, voláteis, metais. No entanto os dados ainda são escassos e insuficientes, gerando poucas informações sobre os efeitos das emissões provenientes deste pólo nos ecossistemas (Lima et al., 2000; Almeida et al. 2003).

A poluição atmosférica é um problema crescente nas grandes cidades. Dados da qualidade do ar, obtidos através de medidas físico-químicas dos níveis de poluição, fornecem informações sobre a presença e a concentração dos poluentes individualmente, porém não são capazes de prever riscos ambientais aos organismos, uma vez que estes reagem à poluição atmosférica e a outros fatores ambientais de maneira integrada (Alves et al., 2001; Lima \& Meira, 2003). A origem deste problema ambiental está na política industrial adotada no país, com um processo de industrialização e urbanização progressivo e descontrolado, acompanhado de um insuficiente controle nas emissões atmosféricas (Lima et al., 2000; Klumpp et al., 2003). O Estado da Bahia não escapa a situação descrita, pois, atualmente, seus ecossistemas encontram-se altamente impactados e descaracterizados, especialmente nos centros urbanos, com alterações na qualidade do ar (Pereira et al., 2007). Nas regiões tropicais e sub-tropicais, pouco se conhece a respeito dos efeitos da influência da poluição atmosférica sobre a estrutura e o funcionamento das espécies vegetais (Klumpp et al., 2000; Carreras et al., 2009); no entanto as respostas de determinadas plantas vêm sendo cada vez mais usadas na valoração quantitativa e qualitativa da contaminação atmosférica e dos riscos ambientais associados (Savóia et al. 2009).

Uma das alternativas para se obter informações de cunho biológico sobre a qualidade do aréatravés do biomonitoramento e/ou bioindicação, que consistem no uso de organismos para verificar e avaliar os efeitos das alterações na composição química, seja do ar, da água ou do solo, tendo como uma das grandes vantagens a detecção de estresse crônico por níveis baixos de poluição agindo por longo tempo (Klumpp, 2001). No entanto, pouco se conhece sobre a relação entre os estressores ambientais e a suscetibilidade dos organismos em responder a esta exposição (Koeman et al., 1993). A utilização de plantas como bioindicadores e/ou biomonitores há muito vem sendo desenvolvida com o objetivo de detectar o impacto e a poluição ambiental causados pelas diversas atividades industriais e urbanas (Wiersma et al., 1980; Sant'Anna et al., 2008), seja em programa de biomonitoramento passivo, quando as espécies já se encontram no ecossistema, ou ativo, quando os organismos são introduzidos (Klumpp, 2001; Oliva \& Figueiredo, 2005). De acordo com Jones \& Heck (1980), as plantas são excelentes monitores biológicos de poluentes atmosféricos, pois apresentam uma sensibilidade relativamente maior em relação aos animais, possuem uma grande variabilidade genética entre e dentro das espécies, o que facilita a seleção do cultivar, da variedade e/ou do clone mais sensível, exibem sintomas foliares característicos de exposição a poluentes. Além disso, por serem imóveis, as plantas permitem uma avaliação mais precisa sobre uma área específica, pois estão fortemente integradas no ambiente em que vivem, discriminando a distribuição espacial do impacto (Lima et al., 2000; Klumpp, 2001). Respostas como injúrias foliares, redução na taxa de fotossíntese, no crescimento, na produtividade, mudanças metabólicas e enzimáticas e a acumulação de elementos tóxicos são alguns dos parâmetros comumente usados em programas de biomonitoramento com plantas (Jones \& Heck, 1980).

Um dos objetivos primários do monitoramento da contaminação atmosférica é identificar onde, quando e a que concentração um poluente ocorre ao longo da fonte de emissão (Divan et al., 2008). Dentre os contaminantes mais perigosos para o ambiente destacam-se os metais devido à alta toxicidade e persistência no ambiente (Oliveira et al., 2001; Carreras et al., 2009) e também o enxofre que provém de processos de combustão (Szabo et al., 2003). Assim, o presente estudo objetiva avaliar o potencial das espécies Anacardium occidentale L., Miconia albicans (Sw.) Triana e Byrsonima sericea $\mathrm{DC}$. como bioacumuladoras de $\mathrm{Al}, \mathrm{As}, \mathrm{Cu}, \mathrm{Ni}, \mathrm{Zn}$ e $\mathrm{S}$ provenientes das atividades industriais do PIC. Como árvores pioneiras, estas espécies contribuem para a fisionomia geral da vegetação na região e são amplamente encontradas em toda a área de estudo.

\section{MATERIAL E MÉTODOS}

\section{Área de estudo}

A área escolhida para o presente estudo situa-se na região do Recôncavo Baiano, sob influência do PIC, e envolve trechos do Distrito de Lamarão do Passé, no Município de São Sebastião do Passé, próximo a uma metalúrgica de cobre, nas seguintes coordenadas geográficas: $12^{\circ} 29^{\prime}$ e $12^{\circ} 40^{\prime} \mathrm{S}$ e $38^{\circ} 18^{\prime}$ e $38^{\circ} 29^{\prime} W$. De acordo com Klumpp et al. (2003) a atividade metalúrgica é responsável por cerca de $20 \%$ da produção de material particulado e $10 \%$ das emissões de $\mathrm{SO}_{2}$. O clima da região, segundo a classificação de Köppen, é definido como As, quente e úmido, sendo mais seco entre os meses de setembro e fevereiro e chuvoso no período de abril a agosto, ocorrendo, esporadicamente, chuvas ao longo da 
estação seca. É caracterizado por possuir elevada temperatura média anual $\left(24^{\circ} \mathrm{C}\right)$, com totais pluviométricos entre 1750 a $2000 \mathrm{~mm}$ anuais. Nesta área manchas de vegetação de restinga, floresta ombrófila densa em seus diversos estádios de desenvolvimento, brejos, mata ciliar e áreas antropizadas são encontradas. Segundo Veloso et al. (1991), a região situa-se no Sistema Edáfico de Primeira Ocupação (Formações Pioneiras), vegetação com influência marinha (restingas), com áreas de ecótono e sistema de vegetação secundária (tratos antrópicos) em segunda fase de sucessão natural. A circulação do vento ocorre, durante o dia, devido à brisa do mar, enquanto à noite esta circulação é invertida, originando uma brisa terrestre, o que embora evite a estagnação atmosférica, atua no transporte dos poluentes em ambas as direções (Lima et al., 2000).

\section{Pontos amostrais e coleta}

Foram estabelecidas cinco áreas de estudo, identificadas de $\mathrm{A} 1$ a A5 (Fig. 1), onde $\mathrm{A} 1$ e A2 são as áreas mais próximas da fonte poluidora e A4 e A5 as mais distantes, a saber:

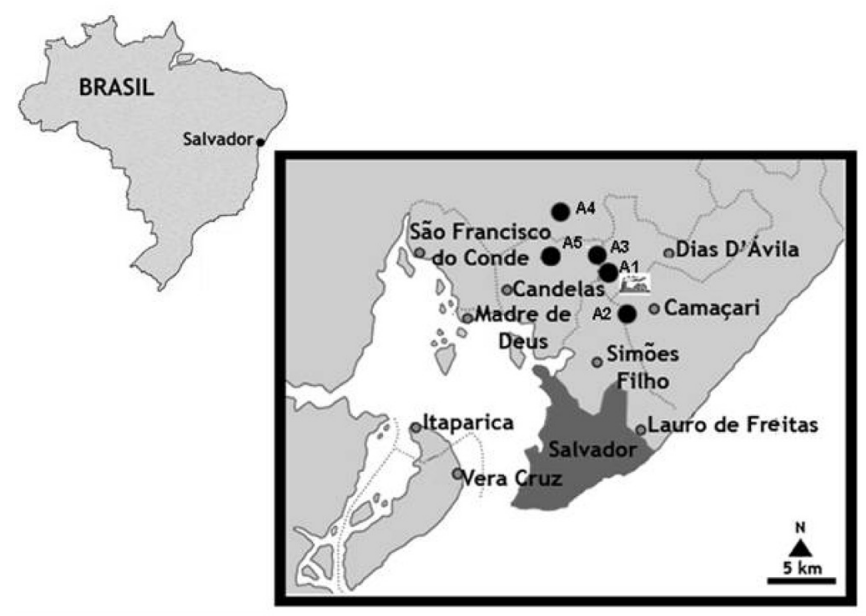

Figura 1 - Áreas de amostragem. A1 - A5 as características das áreas de estudo estão descritas no item material e Métodos.

Área Al (1241'74"S e 38¹9'63"): é uma região que está sujeita às emissões de uma metalúrgica de cobre e de uma indústria de celulose; apresenta reflorestamento com Pinus e Eucalyptus, além de outras espécies introduzidas como Syzigium jambolanum, Clitoria fairchildiana, Terminalia catappa, Joannesia princeps. O solo possui grande quantidade de líter, com camada de material quase totalmente decomposto, com aproximadamente $10 \mathrm{~cm}$ de profundidade. É a área mais próxima da fonte de contaminação.

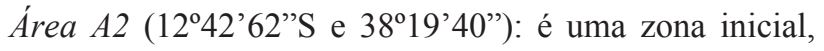
próxima a uma estrada, com uma porção com reflorestamento de Pinus e Eucalyptus, além de espécies introduzidas como Syzigium jambolanum e Clitoria fairchildiana. A outra porção inicial da área, ainda à beira da estrada, representa um grande descampado recoberto por gramíneas. Nesta zona encontra-se também um fragmento de vegetação natural bastante fechada. Está a $3 \mathrm{~km}$ da metalúrgica, porém em sentido oposto à direção predominante do vento.
Área A3 (12 $41^{\prime}$ '08'S e 38²0'46”O): compreende o lado direito da estrada para Lamarão, há uma acentuada degradação. Apresenta solo arenoso, com vestígios de soterramento. Ocorrência predominante de Anacardium occidentale, Tapirira guianensis e Eschweilera ovata. Está a cerca de $5 \mathrm{~km}$ da metalúrgica, no sentido principal do vento, do continente para o mar.

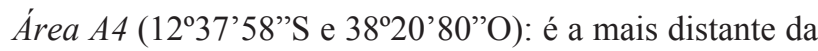
fonte de contaminação $(12 \mathrm{~km})$; está representada por uma vegetação com fisionomia de campos litorâneos, predominando indivíduos de porte arbóreo, com representação expressiva de elementos arbustivos. A vegetação apresenta alguns sinais de devastação.

Área A5 (1240'93''S e 38²2'32”O): compreende uma fazenda situada em Lamarão do Passé, sendo representada por uma mancha de mata ciliar, de natureza ombrófila densa, em meio a uma área de pasto. Neste local, foi encontrado o maior número de espécies de briófitas, em grande parte epífitas, mas com ocorrência de alguns indivíduos terrícolas e epixílicos. Está localizada a, aproximadamente, $8 \mathrm{~km}$ da metalúrgica de cobre, sendo a segunda área mais distante da fonte poluidora.

Em cada área, três indivíduos adultos de cada espécie, A. occidentale (cajueiro), M. albicans (canela de velho) e $B$. sericea (murici da mata), foram, aleatoriamente, selecionados e marcados. A quinta folha de cada ramo, contando da extremidade do ramo para a base, de cada árvore, foi retirada nos quatro pontos cardeais da copa. As folhas foram acondicionadas em sacos plásticos, devidamente preparados, e enviadas para determinação dos teores de $\mathrm{Al}, \mathrm{As}, \mathrm{Cu}, \mathrm{Ni}, \mathrm{Zn}$ and $\mathrm{S}$ em peso seco. As determinações foram feitas utilizandose as metodologias descritas na Tabela 1 .

\section{Tratamento estatístico}

Todos os dados obtidos das análises foliares foram comparados através de análise de variância (ANOVA), sendo realizadas comparações entre as espécies em um mesmo ponto e entre os pontos para uma mesma espécie. Foram aplicados testes de comparação de médias com probabilidade de 95\% - Tukey ou Dunn - para dados paramétricos ou nãoparamétricos, respectivamente (Zar, 1996). Todo o tratamento estatístico foi realizado através do Programa GraphPad Instat versão 3.0 for Windows.

\section{RESULTADOS E DISCUSSÃO}

Os teores de $\mathrm{Al}$ nas folhas das espécies estudadas, nas diferentes áreas, estão na Tabela 2. A maior concentração de $\mathrm{Al}$ em M. albicans foi detectada na área A1 (2.903 mg kg$\left.{ }^{-1}\right)$, porém não houve diferença significativa, nesta espécie, em relação às demais áreas. Os teores de $\mathrm{Al} \mathrm{em} A$. occidentale e B. sericea foram maiores em A2, com concentrações de 49 e $93 \mathrm{mg} \mathrm{Kg}^{-1}$, respectivamente. Para $A$. occidentale, em A2, houve diferença significativa para as demais áreas, exceto para $\mathrm{A} 3$, enquanto para $B$. sericea não foi encontrada 
Tabela 1 - Parâmetro, descrição, método e limites de detecção ( $\mathrm{mg} \mathrm{kg}^{-1}$ ) dos parâmetros inorgânicos em peso seco (PS) utilizados nas determinações inorgânicas.

\begin{tabular}{lccc}
\hline Parâmetro & Descrição & Método & Limite de deteção \\
\hline Alumínio & ICP & $3500-\mathrm{Al}-\mathrm{C}$ & 0,5 \\
Arsênio & ICP & $3500-\mathrm{As}-\mathrm{D}$ & 0,5 \\
Níquel & ICP & $3500-\mathrm{Ni}-\mathrm{C}$ & 1,0 \\
Cobre & AAS & $3500-\mathrm{Cu}-\mathrm{B}$ & 0,5 \\
Zinco & AAS & $3500-\mathrm{Zn}-\mathrm{B}$ & 0,5 \\
Enxofre & Titulação & $80029-\mathrm{TASQA}$ & 5,0 \\
\hline
\end{tabular}

ICP: Espectrometria de emissão óptica com plasma indutivamente acoplado. AAS: Espectrometria de absorção atômica.

Tabela 2 - Teores de Alumínio (mg kg-1) em peso seco (PS) ( $(\mathrm{SD} ; \mathrm{n}=3)$ em folhas de Anacardium occidenale, Byrsonima sericea e Miconia albicans na área do Pólo Industrial de Camaçari.

\begin{tabular}{lccccc}
\hline \multirow{2}{*}{ Espécies } & \multicolumn{4}{c}{ Áreas } \\
\cline { 2 - 6 } & $\mathrm{A} 1$ & $\mathrm{~A} 2$ & $\mathrm{~A} 3$ & $\mathrm{~A} 4$ & $\mathrm{~A} 5$ \\
\hline A. occidentale & $23( \pm 11) \mathrm{Aa}$ & $49( \pm 7) \mathrm{Ab}$ & $30( \pm 10) \mathrm{Aab}$ & $19( \pm 1) \mathrm{Aa}$ & $18( \pm 7) \mathrm{Aa}$ \\
B. sericea & $59( \pm 13) \mathrm{Aa}$ & $93( \pm 28) \mathrm{Aa}$ & $74( \pm 68) \mathrm{Aa}$ & $36( \pm 12) \mathrm{Aa}$ & $54( \pm 30) \mathrm{Aa}$ \\
M. albicans & $2903( \pm 378) \mathrm{Ba}$ & $2533( \pm 397) \mathrm{Ba}$ & $2550( \pm 1062) \mathrm{Ba}$ & $2710( \pm 897) \mathrm{Ba}$ & $2538( \pm 586) \mathrm{Ba}$ \\
\hline
\end{tabular}

As médias seguidas das mesmas letras, maiúsculas nas colunas, e minúsculas nas linhas, não diferem entre si pelo teste de comparação de médias (Tukey ou Dunn), com 5\% de probabilidade. A1 - A5 as características das áreas de estudo estão descritas no item material e Métodos.

diferença entre as cinco áreas. Os dados mostram que $M$. albicans é uma espécie acumuladora de $\mathrm{Al}$, tendo apresentado valores significativamente maiores dos encontrados nas outras espécies em todos os pontos amostrais, chegando a alcançar concentrações duas ordens de magnitude maiores que as outras espécies estudadas. Estes dados corroboram com os de Domingos et al. (1998), em uma floresta da região de Cubatão, São Paulo, onde detectaram que as espécies M. pyrifolia e $M$. саbucu são acumuladoras de poluentes do ar naquela região. Klumpp et al. $(1998,2000)$, detectaram que Tibouchina pulchra (Melastomataceae) também é uma espécie acumuladora de alumínio. Conforme Weinstein \& Davison (2003), muitas espécies de Melastomataceae são geralmente mais tolerantes que sensíveis e essa pode ser considerada uma característica que deve ser levada em consideração na escolha de um biomonitor.

$\mathrm{Na}$ Tabela 3 são apresentados os teores de $\mathrm{Cu}$ nos tecidos foliares das espécies estudadas, nos diferentes locais. Os maiores teores desse elemento foram na área A3 para as espécies A. occidentale e M. albicans, com 71 e $85 \mathrm{mg} \mathrm{kg}$ 1, respectivamente; sem embargo uma grande variação foi observada para ambas. M. albicans mostrou-se mais uma vez acumuladora, sendo a espécie que apresentou maiores teores na maioria das áreas, exceto em A4 e A5, nas quais as maiores acumulações ocorreram em $B$. sericea. Em geral, as espécies apresentaram concentrações estatisticamente semelhantes entre as áreas, exceto entre as concentrações em $A$. occidentale e M. albicans em A3 e A5. Os valores de $\mathrm{Cu}$ aqui encontrados, em algumas áreas, foram maiores dos obtidos por Klumpp et al. (2003), em um estudo no mesmo pólo industrial, no qual observou teores de $\mathrm{Cu}$ em folhas de mangueira (Mangifera indica) de 46,3 e 20,1 $\mu \mathrm{g} \mathrm{g} \mathrm{g}^{-1}$, em duas áreas distantes da metalúrgica de cobre 3 e $5 \mathrm{~km}$, respectivamente. Embora estes mesmos autores tenham encontrado uma intensa redução nos teores de $\mathrm{Cu}$ em folhas de mangueira, à medida que se aumentou a distância da fonte poluidora, demonstrando uma limitada extensão do impacto das emissões, no presente estudo tal gradiente não foi observado. Também neste mesmo complexo industrial, Lima et al. (1997a) constataram que uma maior concentração de cobre no capim-santo (Cymbopongon citratus), resultou em menor taxa de crescimento vegetal, sendo a maior concentração encontrada de 19,13 ppm.

Os maiores teores de $\mathrm{Zn}$ foram encontrados nos tecidos foliares das plantas das áreas A1, A2 e A3, indicando um gradiente com a fonte poluidora (Tabela 4). A concentração mais elevada foi observada para B. sericea em A3 (36 mg $\mathrm{kg}^{-1}$ ), porém não houve diferença significativa para os teores encontrados nas outras espécies nesta mesma área. Esta mesma espécie também apresentou a menor concentração $\left(5,0 \mathrm{mg} \mathrm{kg}^{-1}\right)$ em A5, sendo estatisticamente diferente das concentrações encontradas em $A$. occidenale $\left(7,7 \mathrm{mg} \mathrm{kg}^{-1}\right) \mathrm{e}$ M. albicans $\left(9,7 \mathrm{mg} \mathrm{Kg}^{-1}\right)$. Em geral, semelhante ao ocorrido com as concentrações de $\mathrm{Al}$ e de $\mathrm{Cu}$, não houve uma diferença marcante para uma mesma espécie, entre as áreas.

Para os teores de enxofre observa-se que $M$. albicans mais uma vez mostrou-se uma espécie acumuladora, onde são verificados os valores mais altos encontrados dentre todas as outras (Tabela 5). Em todas as áreas, M. albicans apresentou concentrações significativamente diferentes das detectadas em A. occidentale e B. sericea, exceção ocorrida apenas em A4. Os maiores teores de enxofre em M. albicans foram detectados nas áreas $\mathrm{A} 1$ e A3; entretanto para $A$. occidentale e para $B$. sericea os maiores teores foram observados em A4 e A5. Para todas as espécies não houve diferença estatística significativa quanto à acumulação nas diferentes áreas amostrais. Klumpp et al. (2003) encontraram teores de até $3,8 \mathrm{mg} \mathrm{g}^{-1} \mathrm{em}$ folhas de mangueira, neste mesmo pólo industrial. Para estes autores, a acumulação do enxofre pode ser detectada longe da fonte de emissão, pois este elemento pode ser transportado a longas distâncias, desse modo, esta deve ter sido a razão das espécies terem apresentado teores semelhantes ao longo das diferentes áreas. Conforme Moriarty 
(1999), a proporção de enxofre depositado pode aumentar com a distância da fonte emissora. A constante alteração na direção dos ventos (Lima et al., 2000) pode ser outro fator responsável pela ausência deste gradiente. Os resultados aqui obtidos mostram que o enxofre é acumulado por M. albicans e em menor proporção por $A$. occidentale e $B$. sericea quando expostas à poluição atmosférica. Szabo et al. (2003) observaram relação entre acúmulo foliar de enxofre em uma espécie de Melastomatceae e alterações nos parâmetros de crescimento. Apesar de o enxofre ser um macronutriente essencial para as plantas, um aumento na sua absorção não é positivo, pelo fato de seu composto $\left(\mathrm{SO}_{4}\right)$ ser acídico e precursor de poluentes secundários, que impõem um efeito deletério, causando perdas de outros macronutrientes e distúrbios nutricionais (Moriarty, 1999; Almeida et al., 2003; Szabo et al., 2003). De acordo com Szabo et al. (2003), a maior parte do enxofre absorvido pelas plantas se dá pelas raízes, na forma de sulfato, embora absorção foliar de óxidos de enxofre também pode ocorrer. Indicadores de resposta sobre a exposição ao enxofre, representativos para regiões tropicais, são escassos, para o qual ainda não há um indicador preciso (Klumpp, 2001).

Os elementos As e o Ni também foram monitorados, porém ambos apresentaram valores abaixo do limite de quantificação para todas as espécies em quase todas as áreas (dados não apresentados). A concentração de As, em folhas de mangueira, expostas à poluição do PIC, alcançou valores de até 4,3 $\mu \mathrm{g}$ $\mathrm{g}^{-1}$ (Klumpp, 2001). Lima et al. (1997b), usando Sphagnum sp. como bioacumulador de As no PIC, encontraram valores de 2,2 ppm na estação em Lamarão do Passé e 12 ppm, na estação mais próxima da metalúrgica de cobre. Naquele mesmo estudo, foram observados no coentro (Coriandrum sativum), valores de 0,72 e 0,66 ppm, nas mesmas áreas citadas respectivamente. Isso indica que embora exista uma influência do As e Ni nesta área, as espécies usadas neste estudo não foram capazes de detectá-la.

Parte da acumulação de metais pode estar relacionada com a deposição de material particulado sobre a superfície, a qual pode danificar mecanicamente a superfície da folha além de causar alterações metabólicas. M. albicans exposta no ambiente A1 mostrou sintomas de danos causados pela poluição atmosférica com cloroses e necroses sobre as margens e extremidades das folhas, começando marrom e expandindo das extremidades para a base da folha. Danos moderados foram observados nas folhas das plantas expostas em A4, enquanto danos leves foram registrados nos outros pontos. A fitotoxidade dos elementos atmosféricos e sua influência direta sobre o declínio da floresta na região não estão ainda muito claras. Claramente os compostos fitotóxicos emitidos pelas indústrias instaladas no Pólo Industrial de Camaçari são incorporados à vegetação e estão reagindo com a flora do ecossistema. É possível que a entrada de elementos tóxicos no ecossistema promova trocas no balanço de nutrientes e alterações químicas, ademais dos processos de bioacumulação e danos foliares detectados neste estudo.

Tabela 3 - Teores de Cobre ( $\left.\mathrm{mg} \mathrm{kg}^{-1}\right)$ em peso seco (PS) ( $\left.\pm \mathrm{SD} ; \mathrm{n}=3\right)$ em folhas de Anacardium occidenale, Byrsonima sericea e Miconia albicans na área do Pólo Industrial de Camaçari.

\begin{tabular}{|c|c|c|c|c|c|}
\hline \multirow{2}{*}{ Espécies } & \multicolumn{5}{|c|}{ Áreas } \\
\hline & A1 & A2 & A3 & A4 & A5 \\
\hline A. occidentale & $9,7( \pm 0,6) \mathrm{Aab}$ & $8,6( \pm 0,6) \mathrm{Aab}$ & $71( \pm 67) \mathrm{Aa}$ & $9,0( \pm 1,7) \mathrm{Aab}$ & $5,1( \pm 2) \mathrm{Ab}$ \\
\hline B. sericea & $16( \pm 1) \mathrm{ABa}$ & $14( \pm 3) \mathrm{ABa}$ & $19( \pm 10) \mathrm{Aa}$ & $21( \pm 3) \mathrm{Ba}$ & $15( \pm 2) \mathrm{Ca}$ \\
\hline M. albicans & $21( \pm 4) \mathrm{Bab}$ & $16( \pm 3) \mathrm{Bab}$ & $85( \pm 70) \mathrm{Aa}$ & $17( \pm 6) \mathrm{ABab}$ & $9,3( \pm 0,5) \mathrm{Bb}$ \\
\hline
\end{tabular}

As médias seguidas das mesmas letras, maiúsculas nas colunas, e minúsculas nas linhas, não diferem entre si pelo teste de comparação de médias (Tukey ou Dunn), com 5\% de probabilidade. A1 - A5 as características das áreas de estudo estão descritas no item material e Métodos.

Tabela 4 - Teores de Zinco (mg kg-1) em peso seco (PS) ( $(\mathrm{SD} ; \mathrm{n}=3$ ) em folhas de Anacardium occidenale, Byrsonima sericea e Miconia albicans na área do Pólo Industrial de Camaçari.

\begin{tabular}{|c|c|c|c|c|c|}
\hline \multirow{2}{*}{ Espécies } & \multicolumn{5}{|c|}{ Áreas } \\
\hline & A1 & A2 & A3 & A4 & A5 \\
\hline A. occidentale & $10,4( \pm 0,6) \mathrm{Aab}$ & $12,7( \pm 3,8) \mathrm{Aab}$ & $14,7( \pm 2,1) \mathrm{Ab}$ & $7,7( \pm 1,5) \mathrm{Aa}$ & $7,7( \pm 1,5) \mathrm{Aa}$ \\
\hline B. sericea & $9,3( \pm 0,6) \mathrm{Aab}$ & $13,3( \pm 1,1) \mathrm{Aa}$ & $35,6( \pm 44) \mathrm{Aab}$ & $6,6( \pm 1,1) \mathrm{Aab}$ & $5,0( \pm 0,1) \mathrm{Bb}$ \\
\hline M. albicans & $13,3( \pm 1,2) \mathrm{Ba}$ & $12,7( \pm 1,5) \mathrm{Aa}$ & $12,6( \pm 1,5) \mathrm{Aa}$ & $9,4( \pm 3,1) \mathrm{Aa}$ & $9,7( \pm 0,6) \mathrm{Aa}$ \\
\hline
\end{tabular}

As médias seguidas das mesmas letras, maiúsculas nas colunas, e minúsculas nas linhas, não diferem entre si pelo teste de comparação de médias (Tukey ou Dunn), com 5\% de probabilidade. A1 - A5 as características das áreas de estudo estão descritas no item material e Métodos.

Tabela 5 - Teores de enxofre ( $\left.\mathrm{mg} \mathrm{kg}^{-1}\right)$ em peso seco (PS) $( \pm \mathrm{SD} ; \mathrm{n}=3)$ em folhas de Anacardium occidenale, Byrsonima sericea e Miconia albicans na área do Pólo Industrial de Camaçari.

\begin{tabular}{|c|c|c|c|c|c|}
\hline \multirow{2}{*}{ Espécies } & \multicolumn{5}{|c|}{ Áreas } \\
\hline & A1 & A2 & A3 & A4 & A5 \\
\hline A. occidentale & $124( \pm 66) \mathrm{Aa}$ & $120( \pm 41) \mathrm{Aa}$ & $69( \pm 57) \mathrm{Aa}$ & $131( \pm 65) \mathrm{Aa}$ & $128( \pm 73) \mathrm{Aa}$ \\
\hline B. sericea & $96( \pm 43) \mathrm{Aa}$ & $87( \pm 34) \mathrm{Aa}$ & $115( \pm 57) \mathrm{Aa}$ & $633( \pm 38) \mathrm{Ab}$ & $196( \pm 117) \mathrm{Aa}$ \\
\hline M. albicans & $3086( \pm 1326) \mathrm{Ba}$ & $1103( \pm 662) \mathrm{Ba}$ & $2640( \pm 110) \mathrm{Ba}$ & $1460( \pm 1210) \mathrm{Aa}$ & $1103( \pm 150) \mathrm{Ba}$ \\
\hline
\end{tabular}

As médias seguidas das mesmas letras, maiúsculas nas colunas, e minúsculas nas linhas, não diferem entre si pelo teste de comparação de médias (Tukey ou Dunn), com 5\% de probabilidade. A1 - A5 as características das áreas de estudo estão descritas no item material e Métodos. 


\section{CONCLUSÃO}

Entre as espécies dos ecossistemas estudados, M. albicans mostrou os resultados mais interessantes, demonstrando ser tolerante à poluição do ar, uma característica que pode lhe dar a habilidade para sobreviver em locais altamente poluídos e, como uma espécie pioneira, dominar a fitofisionomia de zonas impactadas, como ocorre nesta região. M. albicans pode ser considerada como uma espécie promissora no monitoramento da contaminação atmosférica na zona estudada devido à capacidade em acumular nas folhas diferentes elementos químicos. As espécies $A$. occidentale e $B$. sericea não acumularam em grandes concentrações os compostos avaliados e tampouco apresentaram sintomas marcantes de injúria foliar.

\section{REFERÊNCIAS}

ALMEIDA, V.S., LIMA, J.S. \& QUEIROZ, A.F.S., 2003, Alterações nos teores de enxofre e metabólitos indicadores de estresse em árvores adultas de Mangifera indica L. CV. Espada expostas à poluição aérea e edáfica, na região do pólo Petroquímico de Camaçari, BA. TECBAHIA 18(1): 82-88.

ALVES, E.S., GIUSTI, P.M., DOMINGOS, M., SALDIVA, P.H.N., GUIMARÃES, E.T. \& LOBO, D.J.A., 2001, Estudo anatômico foliar do clone híbrido 4430 de Tradescantia: alterações decorrentes da poluição aérea urbana. Rev. Bras. Bot. 24(4): $567-$ 576. doi: 10.1590/S0100-84042001000500012

CARRERAS, H.A., WANNAZ, E.D. \& PINGNATA, M.L., 2009, Assessment of human health risk related to metals by the use of biomonitors in the province of Córdoba, Argentina. Environ. Pollut. 157: 117-122. doi:10.1016/j.envpol.2008.07.018

DIVAN, Jr., A.M., OLIVA, M.A. \& FERREIRA, F.A., 2008, Dispersal pattern of airborne emission from an aluminium smelter in Ouro Preto, Brazil, as expressed by foliar fluoride accumulation in eight plant species. Ecol. Indicat. 8: 454-461. doi:10.1016/j.ecolind.2007.04.008

DOMINGOS, M., KLUMPP, A. \& KLUMPP, G., 1998, Air pollution impact on the Atlantic Forest in the Cubatão region, SP, Brazil. Ciência e Cultura 50(4): 230-236.

JONES, H.C. \& HECK, W.W., 1980, Vegetation - Biological indicators or monitors of air pollutants. In: D. L. Worf (ed.). Biological monitoring for environmental effects. Toronto, D.C. Heath and Company, pp. 117-121.

KLUMPP, A., DOMINGOS, M., DE MORAES, R.M. \& KLUMPP, G., 1998, Effect of complex air pollution on tree species of the Atlantic Rain Forest near Cubatão, Brazil. Chemosphere 36(4-5): 989-994. doi:10.1016/S0045-6535(97)10160-6

KLUMPP, G., FURLAN, C.M., DOMINGOS, M. \& KLUMPP, A., 2000, Response of stress indicators and growth parameters of Tibouchina pulchra Cong. exposed to air and soil pollution near the industrial complex of Cubatão, Brazil. Sci. Total Environ. 246: 79-91. doi:10.1016/S0048-9697(99)00453-2

KLUMPP, A., 2001, Utilização de bioindicadores de poluição em condições temperadas e tropicais. In: N.B. Maia; H.L. Martos \& W. Barrella (orgs.). Indicadores ambientais: conceitos e aplicações. São Paulo: EDUC/COMPED/INEP, pp. 77-90.

KLUMPP, A., HINTEMANN, T., LIMA, J.S. \& KANDELER, E., 2003, Bioindication of air pollution effects near a copper smelter in Brazil using mango trees and soil microbiological properties. Environ. Pollut. 126: 313-321. doi:10.1016/S02697491(03)00244-6

KOEMAN, J.H., KÖHLER-GÜNTHER, A., KURELEC, B.,
RIVIÈRE, J.L., VERSTEEG，D. \& WALKER，C.H., 1993, Applications and objectives of biomarker research. In: D.B. Peakall \& L.R. Shugart (eds.). Biomarkers: research and application in the assessment of environmental health. Berlin: Springer-Verlag, pp. 1-13.

LIMA, J.S., CARVALHO FILHO, D.M., COUTO, E., KORN, M.G.A., MELO, M.H. \& GOMES, C.T., 1997a, Capimsanto (Cymbopogon citratus) como bioindicador de poluição atmosférica no Pólo Petroquímico de Camaçari - BA. Rev. Bras. Ecol. 1: 95-98

LIMA, J.S., CARVALHO FILHO, D.M., COUTO, E., SANTANA, D.L. \& SOUZA, H.C., 1997b, Comparação entre o coentro e o Sphagnum sp. como bioacumuladores do arsênio no Pólo Petroquímico de Camaçari - BA. Rev. Bras. Ecol. 1: 91-94.

LIMA, J.S., FERNANDES, E.B. \& FAWCETT, W.N., 2000, Mangifera indica and Phaseolus vulgaris in the bioindication of air pollution in Bahia, Brazil. Ecotoxicol. Environ Saf 46: 275278. doi:10.1006/eesa.1999.1894

LIMA, J.S. \& MEIRA, V.S., 2003, Biomonitoramento da qualidade do ar da cidade do Salvador, BA. TECBAHIA 18(1): 48-54.

MORIARTY, F., 1999, Ecotoxicology - the study of pollutants in ecosystems. Academic Press, USA. 347p.

OLIVA, M.A., FIGUEIREDO, J.G., 2005, Gramíneas bioindicadoras da presença de flúor em regiões tropicais. Revista Brasil. Bot. 28(2): 389-397. doi: 10.1590/S0100-84042005000200017

OLIVEIRA, J.A., CAMBRAIA, J., CANO, M.A.O. \& JORDÃO, C.P., 2001, Absorção e acúmulo de cádmio e seus efeitos sobre o crescimento relativo de plantas de aguapé e de salvínia. R. Bras. Fisiol. Veg. 13(3): 329-341. doi: 10.1590/S010331312001000300008

PEREIRA,P.A.P., LOPES, W.A., CARVALHO, L.S., ROCHA, G.O., BAHIA, N.C., LOYOLA, J., QUITERIO, S.L., ESCALEIRA, V., ARBILLA, J. \& ANDRADE, J.B., 2007, Atmospheric concentrations and dry deposition fluxes on particulate trace metals in Salvador, Bahia, Brazil. Atm. Environ. 41: 7837-7850.

SANT'ANNA, S.M.R., ESPOSITO, M.P., DOMINGOS, M. \& SOUZA, S.R., 2008, Suitability of Nicotiana tabacum 'Bel W3' for biomonitoring ozone in São Paulo, Brazil. Environ. Pollut. 151: 389-394. doi:10.1016/j.envpol.2007.06.013

SAVÓIA, E.J.L., DOMINGOS, M., GUIMARÃES, E.T., BRUMATI, F. \& SALDIVA, P.H.N., 2009, Biomonitoring genotoxic risks under the urban weather conditions and polluted atmosphereic in Santo André, SP, Brazil, through Trad-MCN bioassay. Ecotoxicol. Environ Saf 72: 255-260. doi:10.1016/j. ecoenv.2008.03.019

SZABO, A.V., DOMINGOS, M., RINALDI, M.C.S. \& DELETTI, W.B.C., 2003, Acúmulo foliar de enxofre e suas relações com alterações no crescimento de plantas jovens de Tibouchina pulchra Cogn. (Melastomatacea) expostas na proximidade do pólo industrial de Cubatão, SP. Revista Brasil. Bot. 26(3): 379390. doi: 10.1590/S0100-84042003000300011

VELOSO, H.P., RANGEL FILHO, A.L.R. \& LIMA, J.C.A., 1991, Classificação da vegetação brasileira adaptada a um sistema universal. IBGE. 123 p.

WEINSTEIN, L.H. \& DAVISON, A.W., 2003, Native plant species suitable as bioindicators and biomonitors for airborne fluoride. Environ. Pollut. 125: 3-11. doi:10.1016/S0269-7491(03)00090-3

WIERSMA, G.B., ROGERS, R.C., MCFARLANE, J.C. \& BRADLEY JR., D.V., 1980, Biological monitoring techniques for assessing exposure. In: D.L. Worf(ed.), Biological monitoring for environmental effects. Toronto, D.C. Heath and Company, pp. 123-133.

ZAR, J.H., 1996, Biostatistical Analysis. 3 ed. Prentice-Hall Int, Upper Saddle River, N.J., USA, 662 p. 